\title{
The human medial temporal lobe processes online representations of complex objects
}

\author{
Morgan D. Barense ${ }^{a, *}$, David Gaffan ${ }^{b}$, Kim S. Graham ${ }^{a, c}$ \\ ${ }^{a}$ MRC Cognition and Brain Sciences Unit, 15 Chaucer Road, Cambridge CB2 2EF, United Kingdom \\ ${ }^{\mathrm{b}}$ Department of Experimental Psychology, Oxford University, South Parks Road, Oxford OX1 3UD, United Kingdom \\ ${ }^{\mathrm{c}}$ Wales Institute of Cognitive Neuroscience, School of Psychology, Cardiff University, Tower Building, \\ Park Place, Cardiff CF10 3AT, United Kingdom \\ Received 6 March 2007; received in revised form 24 May 2007; accepted 25 May 2007 \\ Available online 14 June 2007
}

\begin{abstract}
There has been considerable debate as to whether structures in the medial temporal lobe (MTL) support both memory and perception, in particular whether the perirhinal cortex may be involved in the perceptual discrimination of complex objects with a large number of overlapping features. Similar experiments testing the discrimination of blended images have obtained contradictory findings, and it remains possible that reported deficits in object perception are due to subtle learning in controls, but not patients. To address this issue, a series of trial-unique object "oddity" tasks, in which subjects selected the odd stimulus from a visual array, were administered to amnesic patients with either selective bilateral damage to the hippocampus or more extensive damage to MTL regions, including the perirhinal cortex. Whereas patients with damage limited to the hippocampus performed similarly to controls on all conditions, patients with perirhinal damage were significantly impaired when the task required discrimination between objects with a large number of features in common. By contrast, when the same stimuli could be discriminated using simple visual features, patients with perirhinal damage performed normally. These results are consistent with a theoretical view which holds that rostral inferotemporal cortical regions, including perirhinal cortex, represent the complex conjunctions of stimulus features necessary for both perception and memory of objects.
\end{abstract}

(C) 2007 Elsevier Ltd. All rights reserved.

Keywords: Amnesia; Hippocampus; Medial temporal lobe; Perirhinal cortex; Object discrimination; Declarative memory; Perception; Feature conjunctions

\section{Introduction}

Although it is well established that the medial temporal lobe (MTL), a set of heavily interconnected structures including the hippocampus and perirhinal cortex, is essential for the formation of new memories, its role in higher-order perceptual and shortterm memory processes remains controversial (e.g., Barense et al., 2005; Cave \& Squire, 1992; Hannula, Tranel, \& Cohen, 2006; Lee, Bandelow, Schwarzbauer, Henson, \& Graham, 2006; Lee, Buckley et al., 2005, 2006; Lee, Bussey et al., 2005; Levy, Shrager, \& Squire, 2005; Olson, Moore, Stark, \& Chatterjee, 2006; Ranganath \& Blumenfeld, 2005; Shrager, Gold, Hopkins, \& Squire, 2006; Stark \& Squire, 2000). A prominent model of memory proposes an exclusive role for the MTL in long-term

\footnotetext{
* Corresponding author.

E-mail address: morgan.barense@mrc-cbu.cam.ac.uk (M.D. Barense).
}

declarative memory, but not in perception or short-term working memory (Squire, Stark, \& Clark, 2004). Inconsistent with this view, recent investigations have suggested that the perirhinal cortex is critical for the perception of objects with a large number of overlapping features (Barense et al., 2005; Buckley, Booth, Rolls, \& Gaffan, 2001; Bussey, Saksida, \& Murray, 2002; Lee, Buckley et al., 2005; Lee, Bussey et al., 2005). For example, monkeys and humans with perirhinal damage were impaired on concurrent object discriminations with a large number of features in common (termed "feature ambiguity," a property of visual discrimination problems that emerges when features of an object are rewarded when they are part of one object, but not when part of another; Barense et al., 2005; Bussey \& Saksida, 2002; Bussey et al., 2002).

In support of these lesion studies, recent functional neuroimaging studies have provided convergent evidence for a role of the perirhinal cortex in complex object processing. Increased perirhinal cortex activation was reported when participants were 
required to detect an object change across two simultaneously presented grids (Lee, Bandelow et al., 2006), and also during face oddity judgements, in which subjects must determine which of four faces is the odd-one-out from a visual array (Lee, Scahill, \& Graham, 2007b). Activity in the perirhinal cortex has also been observed during naming tasks, in particular when participants were required to name objects at a high (compared to low) degree of specificity, a manipulation thought to stress fine-grained visual discrimination (Tyler et al., 2004). In contrast to the perirhinal cortex, the hippocampus appears to be less involved in either object perception or object item memory (Barense et al., 2005; Baxter \& Murray, 2001; Lee, Buckley et al., 2005; Lee, Bussey et al., 2005; Mayes, Holdstock, Isaac, Hunkin, \& Roberts, 2002; Saksida, Bussey, Buckmaster, \& Murray, 2006), but instead seems critical for processing spatial and/or relational information, even on tasks with only a perceptual or short-term mnemonic demand (Buckley, Charles, Browning, \& Gaffan, 2004; Graham et al., 2006; Hannula et al., 2006; Hartley et al., 2007; Lee, Buckley et al., 2005; Lee, Bussey et al., 2005; Olson, Page, Moore, Chatterjee, \& Verfaellie, 2006; Taylor, Henson, \& Graham, 2007). For example, participants with hippocampal damage have demonstrated impaired processing of spatial scenes (Lee, Buckley et al., 2005; Lee, Bussey et al., 2005), and impaired working memory for the relationship between objects and their spatial locations (Olson, Page et al., 2006). These new findings suggest that the MTL may support processes independent of long-term memory, such as perception and working memory. Furthermore, individual structures within the MTL may be functionally dissociable, with the perirhinal cortex and hippocampus critical for processing complex objects and spatial layouts, respectively.

Two issues, however, limit the interpretation of some of these studies, particularly with respect to the perceptual role of the perirhinal cortex in object processing. First, whereas large deficits following perirhinal damage have been reported for face discrimination (Lee, Buckley et al., 2005; Lee, Bussey et al., 2005), those demonstrated for discrimination of objects are small (Lee, Buckley et al., 2005; Lee, Bussey et al., 2005) or non-existent (Levy et al., 2005; Shrager et al., 2006). Second, when large impairments in object discrimination are reported, the experimental designs involved repetition of the same stimulus set (i.e., the stimuli were not trial unique) (Barense et al., 2005; Lee, Buckley et al., 2005; Lee, Bussey et al., 2005). This leads to the possibility that in these previous studies learning across trials facilitated performance in the controls but not the patients, who may exhibit reduced or delayed learning effects. Although statistical analyses of the performance of the participants included in Lee, Buckley et al. and Lee, Bussey et al. indicated that controls (and patients) did not show any subtle learning effects across trials, a recent study highlighted the importance of using trial-unique stimuli in addressing the role of perirhinal cortex in perception. Using a paradigm similar to that used previously (Lee, Bussey et al., 2005), Shrager et al. (2006) reported normal perceptual discrimination in individuals with perirhinal damage when trial-unique objects and faces were used. This experiment, however, did not systematically manipulate the presence or absence of overlapping object fea- tures (feature ambiguity), a factor that is thought to critically influence performance in participants with perirhinal lesions (Barense et al., 2005). Instead blended images, which typically result in more subtle impairments (Lee, Bussey et al., 2005), were utilized. The current study, therefore, aimed to investigate object processing in humans with MTL damage using a trial-unique design in which feature ambiguity was rigorously controlled. To our knowledge, this study is the first to combine the critical dimension of feature ambiguity within the context of trial-unique discrimination.

\section{Materials and methods}

\subsection{Participants}

Six amnesic patients with focal brain lesions were tested. Based on structural analyses of critical regions within the temporal lobe using an established rating scale validated against volumetric measures (Barense et al., 2005; Galton et al., 2001; Graham et al., 2006; Lee, Bussey et al., 2005) patients were categorized as follows: (1) cases with selective hippocampal damage (hippocampal group; $n=3$ ) and (2) individuals with broader medial temporal lobe damage, including perirhinal cortex (MTL group; $n=3$ ). Of the three patients in the MTL group (age $=69.77$ years; education $=10.33$ years $)$, two were viral encephalitis cases and the third had experienced traumatic intracerebral bleeding. Of the three patients in the hippocampal group (age $=45.69$ years; education $=14.33$ years), one had been diagnosed with viral encephalitis, one had anoxia during status epilepticus, and one experienced carbon monoxide induced hypoxia. For the behavioral experiment, 10 young (age $=48.0$ years; education $=14.4$ years) and 10 elderly (age $=68.38$ years; education $=12.1$ years ) control subjects were age and education matched to the hippocampal and MTL groups, respectively (all $t<1.32$, all $p>0.21$ ).

\subsection{Anatomical analyses}

\subsubsection{Scan rating method}

The visual rating method assessed a total of nine regions, including (1) anterior hippocampus, which was rated on the anteriormost pontine slice and based on the widths of the choroidal fissure and temporal horn and the height of the hippocampal formation; (2) anterior temporal lobe, which was based on the cerebral spinal fluid space between the back of the orbit and temporal pole; (3) amygdala, which was rated on the scan-slice anterior to the tip of the temporal horn; (4) lateral temporal lobe, which was rated on the same slice as the anterior hippocampus and was based on the cortical thickness of the superior and middle temporal gyri; (5) posterior hippocampus, which was rated on the anteriormost slice through the cerebral aqueduct in parallel with the anterior measure and according to the width of the temporal horn and the height of the hippocampal formation; and finally (6) anterior parahippocampal gyrus; (7) medial bank of the collateral sulcus; (8) lateral bank of the collateral sulcus; (9) occipitotemporal sulcus, which were all rated on the slice showing the collateral sulcus at its longest. Other than the anterior hippocampus, which was rated on a five-point scale (normal=0, severe atrophy $=4$; Scheltens et al., 1992), all regions were assessed using a four-point scale (normal $=0$, severe atrophy $=3$ ), with ratings for each area averaged across both hemispheres.

Fig. 1a displays the values obtained from the scan rating method for each individual patient and the mean scores for each of the three subject groups (hippocampal, MTL and control). A repeated-measures ANOVA with a within-group factor of 'region' and a between-group factor of 'subject group' revealed a significant difference in scores across the nine brain areas rated (Greenhouse-Geisser corrected $F(3.8,56.5)=4.84, p<0.01)$, a significant overall difference between the three subject groups $(F(2,15)=75.18, p<0.001)$ and a significant 'region' by 'subject group' interaction (Greenhouse-Geisser corrected $F(7.5,56.5)=3.69$, $p<0.01)$. One-way ANOVAs confirmed a significant group difference on all brain areas (all $F(2,15)>16.4, p<0.001$ ) other than the lateral temporal lobe measure $(F(2,15)=2.28, p>0.14)$. Post hoc analyses on the regions in which there was a significant group difference indicated significantly greater atrophy of 


\begin{tabular}{|l|l|l|l|l|l|l|l|l|l|} 
(a) & AntTemp & Amyg & PHG & MBCS & LBCS & MBOS & AntHC & LatTemp & PostHC \\
\hline HC1 & $1^{*}$ & 1 & $0.75^{*}$ & 0.75 & 0.25 & 0 & $1.5^{*}$ & 1 & 0.75 \\
\hline HC2 & 0 & 0.5 & 0.25 & 0.5 & 0.25 & 0 & $2^{*}$ & 0 & 0.25 \\
\hline HC3 & 0 & 0 & $0.75^{*}$ & 0.75 & 0.5 & 0.25 & $1.25^{*}$ & 0.5 & 1 \\
\hline MTL1 & $2^{*}$ & $2.25^{*}$ & $1.5^{*}$ & $1^{*}$ & $1.25^{*}$ & $2^{*}$ & $1.75^{*}$ & $1.75^{*}$ & $1.75^{*}$ \\
\hline MTL2 & $2^{*}$ & $3^{*}$ & $2.5^{*}$ & $2.75^{*}$ & $2.5^{*}$ & $2^{*}$ & $3^{*}$ & 1 & $2.75^{*}$ \\
\hline MTL3 & $1.75^{*}$ & $2.75^{*}$ & $2.75^{*}$ & $2.75^{*}$ & $2.5^{*}$ & $2.5^{*}$ & $2^{*}$ & 0.5 & $2^{*}$ \\
\hline Control & 0.313 & 0.375 & 0.188 & 0.521 & 0.271 & 0.333 & 0.458 & 0.458 & 0.271 \\
mean & $(0.284)$ & $(0.483)$ & $(0.188)$ & $(0.291)$ & $(0.310)$ & $(0.289)$ & $(0.382)$ & $(0.411)$ & $(0.361)$ \\
\hline
\end{tabular}

(b)

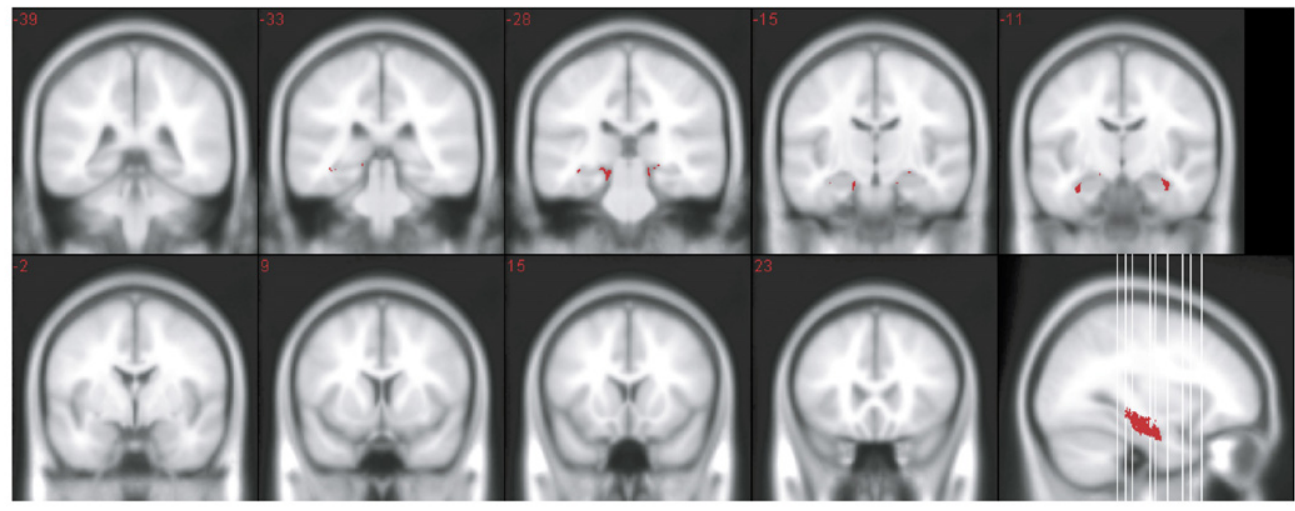

(c)

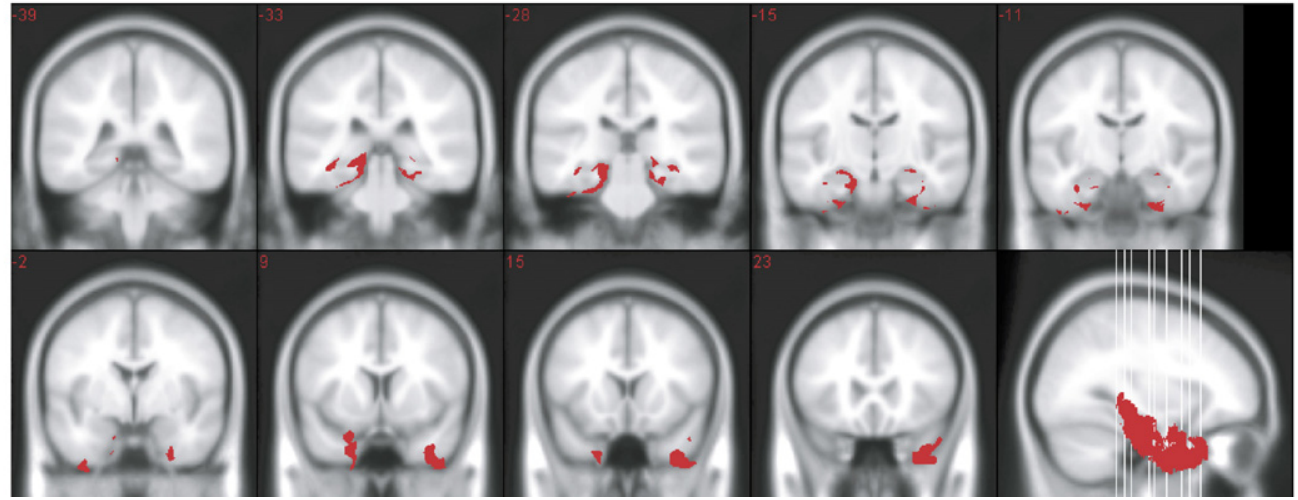

Fig. 1. (a) Structural MRI scan ratings (with standard deviations) for various brain regions (asterisk represents significant difference from controls). 0 indicates no visible damage, 3 (4 for anterior hippocampus) indicates complete absence of area. Ratings for each patient are listed. Abbreviations: AntTemp: anterior temporal cortex; Amyg: amygdala; PHG: parahippocampal gyrus (corresponding to entorhinal cortex); MBCS: medial bank of collateral sulcus (corresponding to the transition between entorhinal and perirhinal cortex); LBCS: lateral bank of collateral sulcus (corresponding to perirhinal cortex); MBOS: medial bank of occipitotemporal sulcus (corresponding to the transition between perirhinal and isocortex); AntHC: anterior hippocampus; LatTemp: lateral temporal cortex (likely to correspond to TE); PostHC: posterior hippocampus. Overlapping regions of damage within the temporal lobe are shown for the (b) hippocampal and (c) MTL groups, superimposed on a Montreal Neurological Institute average brain template.

the anterior hippocampus $(p<0.001)$ and the parahippocampal gyrus $(p<0.05)$ compared to the control group in the hippocampal group, but no other significant differences (all $p>0.1$ ). In contrast, the MTL group received significantly greater rating scores compared to the control group on all measures (all $p<0.001$ ) for which the one-way ANOVAs revealed significant group differences.

As damage lateral to the perirhinal cortex (i.e., area TE) would present a possible confound in the interpretation of our findings, it is important to note that the MTL group, when compared with controls, did not show significant atrophy in our lateral temporal lobe region, with two of three cases possessing no significant damage to this area. Although it is currently unclear exactly what region in the human brain corresponds to area TE in the macaque brain (Von Bonin \& Bailey, 1947; Seltzer \& Pandya, 1978), area TE in macaques is known to occupy the inferior and middle temporal gyri, the latter of which was included in the lateral temporal lobe rating. Consistent with this, the profile of performance in the MTL group did not match existing knowledge of the effects of damage to area TE in nonhuman primates. All three MTL cases demonstrated normal color discrimination (see Section 3), a process thought to be dependent on lateral temporal areas in macaque monkeys (Horel, 1994; Buckley \& Gaffan, 1997). Furthermore, as discussed in more detail in Section
3.1, the performance of the patient with lower than normal lateral cortex volume (MTL1) was indistinguishable from the other two MTL cases, indicating that damage to the lateral temporal lobe is unlikely to account for the observed deficits.

\subsubsection{MRIcro analysis}

In addition to this rating scale, regions of atrophy within the temporal lobe were delineated for the three hippocampal patients and two of the three patients in the MTL group (an electronic version of the scan for the individual classified as MTL2 in Fig. 1a was not available) using MRIcro (Rorden \& Brett, 2000). The structural scans were first warped into Montreal Neurological Institute (MNI) space in SPM99 (Wellcome Department of Functional Neuroscience, London, UK) using a standard procedure for brain images with focal lesions (Brett, Leff, Rorden, \& Ashburner, 2001). This involved creating a mask in MRIcro for each of the subjects' lesions by delineating regions of cerebral spinal fluid in the middle cranial fossae, including the inferior horn and choroidal fissure, until the posterior limit of the end of the hippocampus was reached. These masks were then used for cost function masked normalization of each brain to a standard T1 MNI template. Following warping, the lesions of each patient were then 
redrawn and finally, overlaid onto an average brain T1 MNI template using MRIcro. Overlapping regions of damage within the temporal lobe are shown for each patient group in Fig. 1b and c. The region of overlapping damage across all three patients classified in the hippocampal group was limited to the hippocampus bilaterally. By contrast, the MTL patients had broader MTL damage encompassing the hippocampus and the perirhinal cortex. Fig. 1c shows that there was an increased amount of cerebral spinal fluid in the region of the collateral sulcus and corresponding to the ventromedial aspect of the temporal pole, in line with recent descriptions of the perirhinal cortex (Davies, Graham, Xuereb, Williams, \& Hodges, 2004; Insausti et al., 1998; Suzuki \& Amaral, 1994).

\subsection{Neuropsychological battery}

The patients were administered a series of standardized neuropsychological tests to assess their memory and visual perception (results reported in Table 1). In summary, these demonstrated that both groups of patients had deficits in episodic memory and were equally impaired on recall-based memory tests. For instance, both patient groups performed poorly on measures of episodic recall, such as the Logical Memory (WMS-III, Story 1 and 2) immediate recall, delayed recall, recognition and on delayed recall of the Rey Complex Figure. Independent samples $t$-tests revealed no significant differences between the two groups on these tests $(p=0.35,0.34,0.46$, and 0.34 , respectively). In addition, the two patient groups were matched in their performance on the Warrington Recognition Memory Test for Words $(p=0.11)$. By contrast, there was a significant difference between the two groups on the Warrington Recognition Memory Test for Faces $(p<0.001)$, with normal performance in the hippocampal group but a significant deficit in the MTL group. Visuoperceptual performance as measured by the Benton Facial Recognition Test, Rey Complex Figure Copy and Visual Object Space Perception Battery (both groups passed all object and space tests) was within the normal control range on all tasks, although comparisons between the two groups revealed a significant difference on the Benton Facial Recognition Test (MTL group impaired relative to hippocampal group, $p<0.05$ ), but not on the Rey Complex Figure Copy $(p=0.20)$. It is important to emphasize, however, that these measures are not sufficiently taxing to reveal the perceptual deficits of the type previously observed in these patients (Lee, Buckley et al., 2005; Lee, Bussey et al., 2005). Whereas the hippocampal group exhibited intact semantic function, the MTL group was mildly impaired on semantic memory, including Word-Picture Matching $(p<0.01)$, naming $(p=0.07)$ and the Pyramids and Palm Trees Test (pictures) $(p<0.05)$. Both groups performed in the normal range on executive tasks (Wisconsin Card Sorting; forwards and backwards digit span; Tower of London; Raven's Colored Progressive Matrices). Independent samples $t$-tests revealed no significant differences between the two groups on these tests $(p=0.50,0.73,0.64,0.14,0.15$, respectively).

\subsection{Behavioral procedure}

All tasks were based on an oddity paradigm in which the subjects were instructed to select the "odd-one-out" from an array of simultaneously presented stimuli, as quickly but as accurately as possible (e.g., Buckley et al., 2001; Lee, Buckley et al., 2005). During the test, touching any item on the touchscreen resulted in the offset of the stimulus display. No feedback was given. Both accuracy and response times were recorded. The patients were tested in their own homes, and control subjects were tested at the MRC Cognition and Brain Sciences Unit. All tests were computerized tasks and were conducted on a $15^{\prime \prime}$ SVGA LCD touchscreen at $1024 \times 768$ resolution. The experiments were programmed using E-Prime software (Psychology Software Tools Inc., Pittsburgh, PA). Subjects were seated in front of the screen so that they could comfortably touch it. All participants gave informed consent before undertaking the study. This investigation received ethical approval from the Cambridge and Southampton Health Authority Local Research Ethics Committees (UK).

To avoid any confounding effects of memory for previously viewed stimuli, all the items for each of the nine conditions reported below were trial-unique. Any patient deficits on these tasks are therefore unlikely to be explained by difficulties in learning individual stimuli across trials.

\subsection{Experiment 1: fribbles}

Participants were presented with an array of seven stimuli and were asked to identify the object that did not contain an identical pair in the array (i.e., in each array, there were three sets of "identical twins" and one odd-one-out). This adaptation of the typical oddity paradigm reported in previous studies (e.g. Buckley et al., 2001; Lee, Buckley et al., 2005) allowed for the systematic manipulation of feature ambiguity across objects. The stimuli were "fribbles" (Williams \& Simons, 2000), novel objects composed of a main body and four appendages (see Fig. 2a). There were 12 categories (or "species") of fribbles in total. Within a species, all fribbles consisted of the same main body, but each of the four appendages had three possible values. Thus, there were 81 unique exemplars within each species.

As demonstrated in Fig. 2b-d, each trial could be one of three different levels of perceptual discrimination: (1) minimum feature ambiguity (all features were unique to the odd-one-out and paired items), (2) intermediate feature ambiguity (two features were held constant across all fribbles while the remaining two appendages maximally overlapped across all fribbles), and (3) maximum feature ambiguity, in which all appendages were maximally overlapping (i.e., every feature was present on either three or four fribbles). Thus, in both the maximum and the intermediate condition, it was only the conjunction of two features that correctly distinguished the odd-one-out from the remaining pairs. By contrast, the minimum ambiguity condition could be solved on the basis of a single feature (i.e., by comparing one appendage).

A short practice of five easy trials (two trials that consisted of letters only to convey the concept of the task, and three trials using fribbles from different species with different bodies) was given before commencing the experiment. The actual experiment consisted of 108 trials, split into two blocks of 54 trials each with a short break between blocks. The different trial types were randomized within a block and an equal number of trials from the minimum, intermediate and maximum trials were given (i.e., 36 trials per condition). Each fribble species was equally represented across the different ambiguity levels. The position of the odd-one-out was counterbalanced, and the positions of the six-paired items were randomly determined. The trials were then checked and altered to ensure that no trial had two sets of identical fribbles adjacent to one another.

\subsection{Experiment 2: four-choice oddity}

As the intermediate and maximum ambiguity conditions in Experiment 1 were significantly more difficult than the minimum condition for controls (all $p<0.05$, see Fig. 3a), six oddity tasks with only four items presented per trial (similar oddity paradigm to that reported previously, Buckley et al., 2001; Lee, Buckley et al., 2005) were used in Experiment 2 (Fig. 2e-j). Six oddity tasks were administered: low ambiguity familiar objects, high ambiguity familiar objects, low ambiguity greebles, high ambiguity greebles, size and color. In all tests, subjects were presented with four stimuli on a white background, three of which were identical and one of which was different. The four familiar object and greeble tasks were presented in a counterbalanced order across all subjects. The size and color tasks were always presented after these four conditions.

Thirty-five trials were administered for the familiar object, greeble and size tasks, and 65 trials for the color task. The order of the trials was fully randomized, and the position of the odd-one-out was counterbalanced across trials.

\subsection{Greeble tasks}

Four pictures of "greebles" (e.g., Gauthier \& Tarr, 1997) were presented for each trial. Each greeble was rotated either $0^{\circ}, 90^{\circ}, 180^{\circ}$, or $270^{\circ}$ from the upright position. There were two available pictures taken from different views of each greeble. Thus, within each trial, there were three foils (the greeble from view 1 and view 2, and a duplication of either view 1 or 2) and one odd-one-out. Whether the odd-one-out and duplicated greeble were shown from view 1 or 2 was fully counterbalanced.

Within the greeble tasks, there were two different conditions: high and low ambiguity (Fig. 2e and f). The criteria for the high ambiguity task were that the greebles be from the same family, the same gender, and be of the same symmetry (i.e., asymmetrical versus symmetrical). Within those criteria, the greebles for each trial were selected to produce the maximum amount of possible feature 
Table 1

Scores for each patient on a range of neuropsychological tests

\begin{tabular}{|c|c|c|c|c|c|c|c|c|c|c|}
\hline & $\mathrm{HC} 1$ & $\mathrm{HC} 2$ & $\mathrm{HC} 3$ & $\begin{array}{l}\text { HC mean } \\
\text { raw score }\end{array}$ & $\mathrm{HC} \%$ score & MTL1 & MTL2 & MTL3 & $\begin{array}{l}\text { MTL mean } \\
\text { raw score }\end{array}$ & $\begin{array}{l}\text { MTL \% } \\
\text { score }\end{array}$ \\
\hline Etiology & Anoxia & Viral encephalitis & $\mathrm{CO}$ poisoning & & & Traumatic intracerebral bleeding & Viral encephalitis & Viral encephalitis & & \\
\hline \multicolumn{11}{|l|}{ Recall } \\
\hline $\begin{array}{l}\text { WMS III immediate story } \\
\text { recall (75) }\end{array}$ & 21.0 & 31.0 & 22.0 & 24.7 & 32.9 & 12.0 & 29.0 & 13.0 & 18.0 & 24.0 \\
\hline $\begin{array}{l}\text { WMS III delayed story } \\
\text { recall }(50)\end{array}$ & 4.0 & 24.0 & 4.0 & 10.7 & 21.3 & 3.0 & 0.0 & 4.0 & 2.3 & 4.7 \\
\hline RCF delayed recall (36) & 6.5 & 18.0 & 3.0 & 9.2 & 25.5 & 7.0 & 0.0 & 4.5 & 3.8 & 10.6 \\
\hline \multicolumn{11}{|l|}{ Recognition } \\
\hline $\begin{array}{l}\text { WMS III story recognition } \\
(30)\end{array}$ & 23.0 & 24.0 & 19.0 & 22.0 & 73.3 & 19.0 & 19.0 & 23.0 & 20.3 & 67.8 \\
\hline WRMT words (50) & 36.0 & 42.0 & 33.0 & 37.0 & 74.0 & 19.0 & 31.0 & 31.0 & 27.0 & 54.0 \\
\hline WRMT faces $(50)$ & 48.0 & 48.0 & 44.0 & 46.7 & 93.3 & 32.0 & 32.0 & 30.0 & 31.3 & 62.7 \\
\hline \multicolumn{11}{|l|}{ Visuoperceptual } \\
\hline Rey copy (36) & 36.0 & 36.0 & 35.0 & 35.7 & 99.1 & 33.0 & 36.0 & 30.5 & 33.2 & 92.1 \\
\hline $\begin{array}{l}\text { Benton Facial Recognition } \\
\text { Test (54) }\end{array}$ & 49.0 & 46.0 & 47.0 & 47.3 & 87.7 & 41.0 & 45.0 & 42.0 & 42.7 & 79.0 \\
\hline VOSP (all sub-tests) & Pass & Pass & Pass & - & & Pass & Pass & Pass & & \\
\hline \multicolumn{11}{|l|}{ Semantic } \\
\hline Naming (64) & 63.0 & 62.0 & 64.0 & 63.0 & 98.4 & 28.0 & 55.0 & 46.0 & 43.0 & 67.2 \\
\hline $\begin{array}{l}\text { Word-picture matching } \\
\text { (64) }\end{array}$ & 64.0 & 64.0 & 64.0 & 64.0 & 100.0 & 57.0 & 59.0 & 54.0 & 56.7 & 88.5 \\
\hline PPT pictures (52) & 51.0 & 51.0 & 52.0 & 51.3 & 98.7 & 45.0 & 49.0 & 46.0 & 46.7 & 89.7 \\
\hline \multicolumn{11}{|l|}{ Executive } \\
\hline WCST (categories, 6) & 5.0 & 6.0 & 6.0 & 5.7 & 94.4 & n.t. & 6.0 & 6.0 & 6.0 & 100.0 \\
\hline Digit span—forwards & 8.0 & 6.0 & 6.0 & 6.7 & - & 7.0 & 8.0 & 6.0 & 7.0 & - \\
\hline Digit span—backwards & 7.0 & 4.0 & 6.0 & 5.7 & - & 4.0 & 7.0 & 4.0 & 5.0 & - \\
\hline $\begin{array}{l}\text { TOL (correct solutions, } \\
\text { 16) }\end{array}$ & 15.0 & 16.0 & 16.0 & 15.7 & 97.9 & 11.0 & 13.0 & n.t. & 12.0 & 75.0 \\
\hline RCPM (36) & 35.0 & 34.0 & 34.0 & 34.3 & 95.4 & 19.0 & 33.0 & 22.0 & 24.7 & 68.5 \\
\hline
\end{tabular}

Maximum scores are provided in brackets where applicable. Individual cells for each patient represent raw data scores; \% score reflects the average percentage score for each group. Abbreviations: WMS III: Wechsler Memory Scale, third edition; Rey: Rey Complex Figure (Osterrieth, 1944); WRMT: Warrington Recognition Memory Test (Warrington, 1984); VOSP: Visual Object and Space Perception Battery (Warrington \&

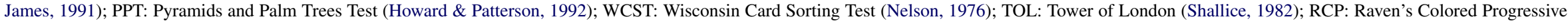
Matrices (Raven, 1962); n.t. = not tested. 
(a)

Minimum Ambiguity:

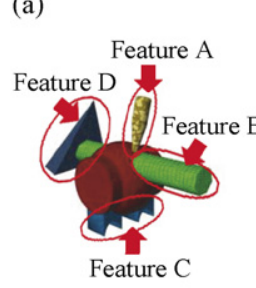

ABCD EFGH ABCD EFGH

JKLM NOPQ JKLM

Intermediate Ambiguity:

ABCE ABCE ABDF ABDE

ABDF ABCF ABDE

Maximum Ambiguity:

ACEG BDEG ACEG ADFH

ADFH BCFH BDEG
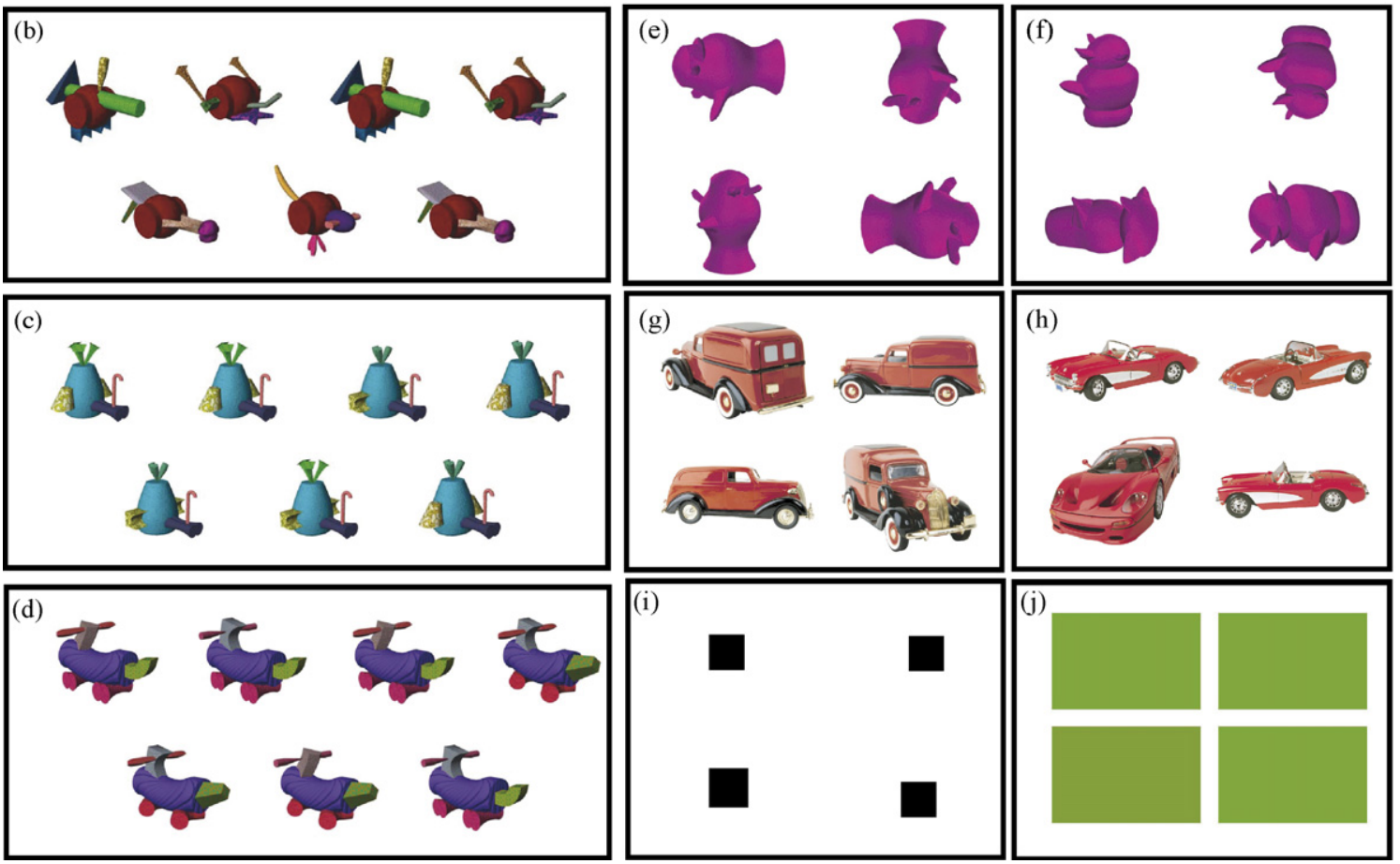

Fig. 2. Object oddity tasks. (a) A fribble is composed of a central body and four attached features. The overlap of these features across fribbles was varied according to the letter schematic (the correct answer (i.e., the fribble without an identical pair) is shown in red). Representative trials from the minimum (b), intermediate (c) and maximum (d) conditions in Experiment 1. Representative trials from Experiment 2 (correct answer is located in bottom left corner): (e) high (greebles from same family and gender) and (f) low (greebles from different families) ambiguity greebles, high (g) and low (h) ambiguity familiar objects, size (i) and color (j).

overlap between the odd-one-out and the foils. The criterion for the low ambiguity task was that the greebles be from different families. The greebles could be either the same or different gender, and be of the same or different symmetry. The different combinations of symmetry and genders were fully counterbalanced across trials.

\subsection{Familiar object tasks}

Four images of objects common to everyday life were presented in each trial, and each photograph was taken from four different non-specific orientations. Objects were collected from the Hemera Photo-Objects Image Collection (Volumes 1-3). As with the greeble tasks, there were two different conditions: high ambiguity and low ambiguity (Fig. $2 \mathrm{~g}$ and h). Unlike in the fribbles and greebles conditions, the level of ambiguity was determined subjectively, with extreme care taken to ensure that within a high ambiguity trial, the two objects shared a high number of overlapping features. By contrast, within a low ambiguity trial, the two objects were from the same overall category (e.g., cars) but the two objects were easily differentiated on the basis of a single, obvious feature. Furthermore, the stimulus types were matched across the low and high ambiguity conditions (e.g., there was a high and a low trial comprised of cars, a high and a low trial comprised of stereos, etc).

\subsection{Size}

Four black squares were presented in each trial (Fig. 2i). The length of each side was randomly varied from 67 to 247 pixels, and the size of each square was trial-unique. In each trial, either three identical smaller squares were shown with one larger square or three identical larger squares were shown with one smaller square. The difference between the two sizes varied between 9 and 15 pixels. The positions of squares were jittered slightly so that the edges did not line up along vertical or horizontal planes.

\subsection{Color}

Four colored squares of dimensions $425 \times 275$ pixels were shown for each trial (Fig. 2j). In each trial, three squares were an identical color, and one was a different color. To generate the colors, the proportion of green and red that each color contained was varied, and the "blue" dimension was held constant at $0 \%$. Each color was trial-unique and luminance was equated across all four squares.

Depending on the willingness of each individual participant, Experiments 1 and 2 were either administered in the same session or in different sessions separated by approximately 1 week. Half of the participants performed Experiment 1 prior to Experiment 2, and vice versa.

\section{Results}

Repeated-measures analyses of variance (ANOVAs) were conducted on the accuracy scores (as measured by percent 

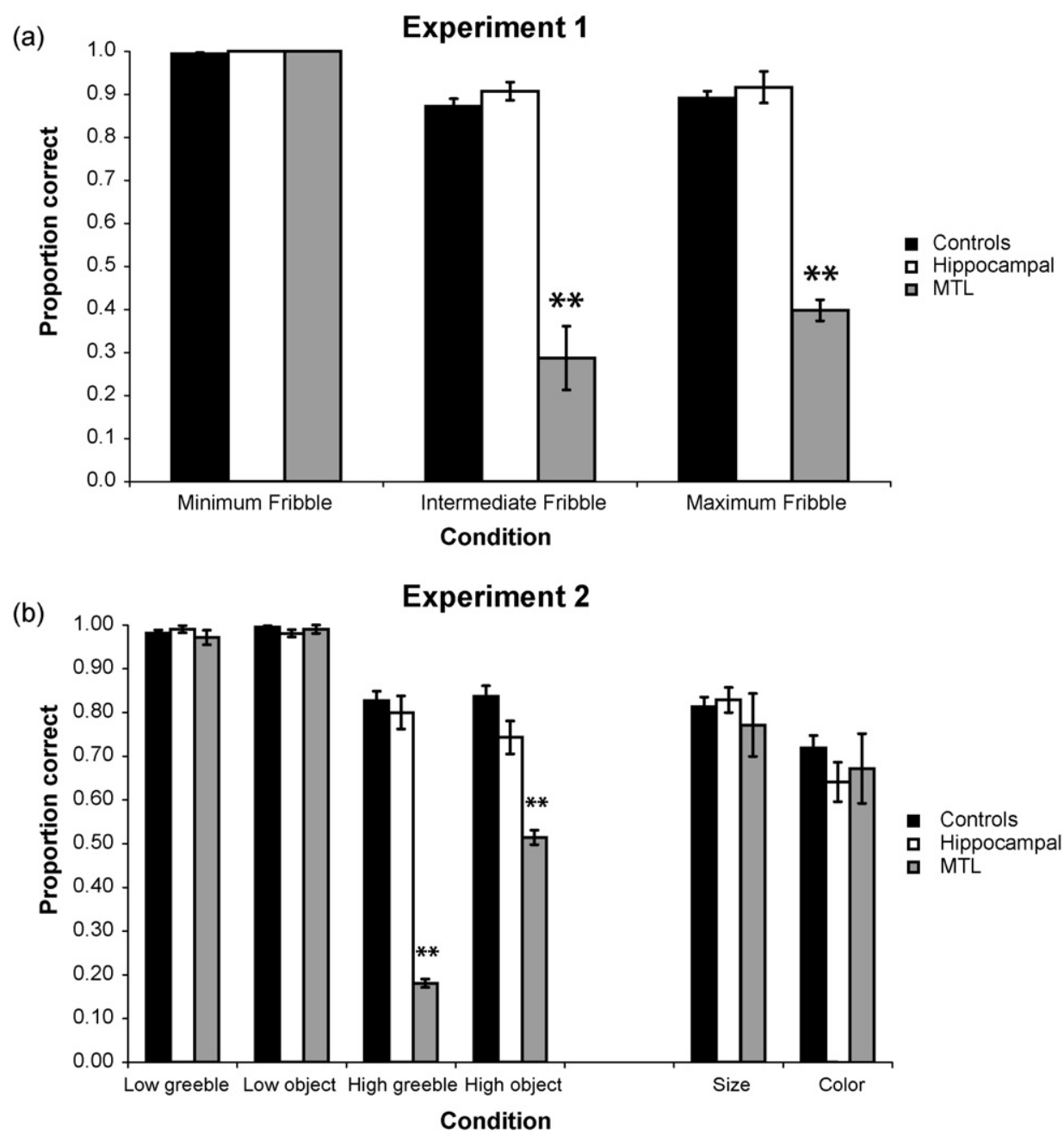

Fig. 3. Mean proportion correct for the two patient groups and two control groups (averaged) for Experiment 1 (a) and Experiment 2 (b). Error bars represent S.E.M. $* * p<0.001$.

correct). ${ }^{1}$ The performance accuracy data from Experiment 1 (Fig. 3a) were subjected to a repeated-measures ANOVA with a within-subject factor of 'ambiguity' (i.e., minimum, intermediate, and maximum) and two between subject factors of 'health' (i.e., patient versus control) and 'lesion type' (i.e., hippocampal (patients and controls) versus MTL (patients and controls)). In Experiment 2, to assess the effects of (1) novel versus familiar objects and (2) high versus low ambiguity on performance of the four choice oddity conditions, the performance accuracy data (Fig. 3b) were subjected to a repeated-measures ANOVA. The

\footnotetext{
${ }^{1}$ Although the ANOVAs performed did not require adjustments for nonsphericity as assessed by Mauchly's $W$ statistic, given the small patient sample sizes, we also conducted non-parametric tests (Mann-Whitney statistics). Importantly, these revealed an identical pattern of performance to that described by the parametric tests. The hippocampal group was unimpaired relative to their control group on all discrimination tests (all $p>0.11$ ). By contrast, the MTL cases were significantly impaired relative to their control group on discriminations involving ambiguous features (i.e., intermediate and maximum fribbles, high ambiguity greebles and objects; all $p<0.01$ ), but demonstrated normal performance on discriminations which could be solved on the basis of a single feature (i.e., minimum fribbles, size, color, low ambiguity greebles and objects; all $p>0.43$ )
}

same between-subject factors of 'health' and 'lesion type' were incorporated, along with two within-subject factors of 'ambiguity', with two levels corresponding to the ambiguity of the stimuli (i.e., low versus high), and 'familiarity', with two levels corresponding to the familiarity of the stimuli (i.e., novel greebles versus familiar objects). In both experiments significant interactions were investigated further using univariate ANOVAs and $t$-tests. As a variable of interest (feature ambiguity) was consistent across both Experiments 1 and 2, the results for these two experiments are described together below. Additional analyses on the accuracy data from Experiment 2 (i.e., the effect of familiarity and performance on the size and color conditions) are described separately.

\subsection{Effect of feature ambiguity}

Repeated-measures ANOVAs demonstrated that in both experiments, the interactions between health and lesion type were significant $(F(1,22)=42.05, p<0.001$ and $F(1$, $22)=24.14, p<0.001$, in Experiments 1 and 2, respectively). In addition, the three-way interaction between health, lesion type and ambiguity was significant across both experiments, 
indicating that the two patient groups performed differently relative to their respective controls as ambiguity increased $(F(2$, $44)=27.80, p<0.001$ and $F(1,22)=25.95, p<0.001$, for Experiments 1 and 2, respectively). Univariate ANOVAs on the performance data from each individual task revealed significant interactions between health and lesion type on the intermediate and maximum feature ambiguity conditions in Experiment 1 and on the object and greeble high ambiguity conditions in Experiment 2 (all $F>5.8$, all $p<0.05$ ). Independent samples $t$-tests demonstrated that the MTL group was impaired relative to their controls on all these conditions (all $t>7.1$, all $p<0.001$ ), whereas the hippocampal group was not (all $t<1.6$, all $p>0.15$ ). By contrast, univariate ANOVAs showed no significant interaction between health and lesion type on single feature discriminations (minimum feature ambiguity (Experiment 1), object and greeble low ambiguity (Experiment 2); all $F<1.2$, all $p>0.27$ ), indicating that both patient groups performed normally relative to their controls on these conditions.

It is also noteworthy that the performance across the subjects in the MTL group was nearly identical. For example, within each maximum ambiguity condition across both experiments, the subjects in the MTL group committed within three errors of one another. This remarkably narrow range of performance in the MTL group indicates that the individual with more lateral damage (Patient MTL1, see Table 1 and Fig. 1a) did not drive the effects reported in the present study.

\subsection{Effect of stimulus familiarity (novel greebles versus familiar objects)}

The four-way interaction between health, lesion type, ambiguity and familiarity was also significant in Experiment 2 $(F(1,22)=9.60, p<0.01)$ due to a two-way interaction between familiarity and ambiguity in the MTL group $(F(1,2)=363.66$, $p<0.01$ ), but not in the other three groups (all $F<1.42$, all $p>0.26$ ). Paired samples $t$-tests revealed poorer performance on high ambiguity greebles compared to high ambiguity objects in the MTL group $(t(2)=34.97, p<0.001)$, but not in the other three subject groups (all $p>0.34$ ). Performance of the MTL group was matched on the low conditions of both stimulus types $t(2)=1.0, p=0.42$. Thus, the MTL group demonstrated worse performance on high novel ambiguity discriminations compared to high familiar ambiguity discriminations.

\subsection{Control tasks: color and size}

The performance accuracy data (shown as proportion correct in Fig. 3b) for the four subject groups on the control tasks (color and size used in Experiment 2) were subjected to a repeatedmeasures ANOVA. A single within-subject factor of 'task' was incorporated with two levels corresponding to the type of oddity task (i.e., color versus size). The same two between-subject factors of health and lesion type were included. This analysis showed no significant effect of health $(F(1,22)=1.48, p=0.24)$, no significant interaction between health and lesion type $(F(1$, $22)=0.44, p=0.51$, and no interaction between health, lesion type and task $(F(1,22)=0.55, p=0.46)$, indicating that the hip- pocampal and MTL groups both performed similarly to their controls.

\subsection{Difficulty analyses}

To investigate the issue of task difficulty, the performance data from all control participants included in Experiments 1 and 2 were subjected to a repeated-measures ANOVA. A single within-subject factor of 'task' with nine levels (i.e., minimum, intermediate and maximum ambiguity fribbles, low ambiguity greebles and objects, high ambiguity greebles and objects, color and size) was included. As only control performance was investigated, there was no between-subject factor. This analysis showed a significant effect of task $(F(8,152)=28.33, p<0.001)$. Post hoc analyses (Bonferroni-corrected $t$-tests) were performed and the most meaningful of the 36 possible comparisons are reported here. In Experiment 1, the minimum ambiguity condition was less difficult than the intermediate and maximum ambiguity conditions (all $p<0.05$ ), but the latter two conditions were matched for difficulty $(p=1.0)$. Critically, in Experiment 2 control performance indicated that the size and color control tasks were either as difficult (all $p>0.34$ ) or more difficult (color versus high ambiguity object, $p<0.05$ ) than the high ambiguity greeble and object conditions. Performance on the high object and greeble conditions was matched $(p=1.00)$. Comparison of performance on conditions across the two experiments revealed that the intermediate and maximum fribbles were matched in difficulty to the size and high ambiguity greeble and object discriminations ( $p=0.06$ for maximum fribbles versus high ambiguity objects, all other $p>0.11$ ), but were less difficult than the color discriminations (all $p<0.01$ ). Thus, given that controls found the control tasks (size and color oddity) to be as or more difficult than the high ambiguity conditions, the discrimination deficits present in the patients cannot easily be attributed to differences in task difficulty.

\subsection{Reaction time analyses across Experiments 1 and 2}

An identical series of analyses to those conducted on the accuracy data were performed on the reaction time data. Reaction times for correct responses only were included (the outcome of the analyses does not change if reaction times for both correct and incorrect responses are included). These analyses revealed no significant differences between the subject groups. The interaction between 'health' and 'lesion type' was not significant in either experiment $(F(1,22)=1.80 ; p=0.19$ and $F(1,22)=0.021$; $p=0.89$, in Experiments 1 and 2, respectively). The three-way interaction between health, lesion type and 'ambiguity' (i.e., minimum, intermediate and maximum conditions in Experiment 1, and low versus high conditions in Experiment 2) was also not significant in either experiment, indicating that the two patient groups performed similarly relative to their respective controls as ambiguity increased $(F(2,44)=2.37 ; p=0.11$ and $F(1,22)=0.097 ; p=0.76$, for Experiments 1 and 2, respectively). In addition, analyses on the reaction time data from the control color and size tasks revealed no differences across groups. This analysis showed no significant interaction between 
health and lesion type $(F(1,22)=0.54 ; p=0.47)$, and no interaction between health, lesion type and task $(F(1,22)=1.52$; $p=0.23$ ). Thus, in summary, the hippocampal and MTL groups both performed all discriminations as quickly as their controls, but - as confirmed by the accuracy analyses - the MTL group committed significantly more errors.

\section{Discussion}

Three patients with MTL damage that included the perirhinal cortex performed normally on object discriminations which could be solved on the basis of a single feature, but were severely impaired if the task stressed feature ambiguity by requiring discrimination of conjunctions of object features. This pattern was observed for discriminations of both novel and familiar stimuli, with a greater deficit for novel discriminations. Notably, poor performance was evident in two experiments in which trialunique stimuli were utilized, a finding counter to the argument that impairments in object processing after perirhinal cortex lesions result from deficient learning across trials with repeated stimuli (Squire, Shrager, \& Levy, 2006; Squire et al., 2004). Furthermore, these deficits cannot be explained by a simple difficulty differential between the low and high ambiguity conditions, as patients were unimpaired on two control oddity tasks (size and color) that controls found as difficult as the critical experimental conditions. Highlighting the specialized role of non-hippocampal MTL regions in object discrimination, participants with hippocampal damage performed normally on all conditions regardless of ambiguity or difficulty, both in terms of accuracy and reaction times.

Our preferred explanation for the data presented here is that the perirhinal cortex is responsible for storing and processing representations of complex, feature conjunctive object stimuli, a conclusion consistent with neuropsychological studies showing deficits in face processing after MTL damage that involves the perirhinal cortex (Lee, Buckley et al., 2005; Lee, Bussey et al., 2005) and functional neuroimaging reports of selective perirhinal cortex recruitment during object-based processing (Lee, Bandelow et al., 2006). These findings broaden the role of the MTL beyond long-term memory processing and support a representational account of MTL function. Under this theory, rostral inferotemporal cortical regions, including perirhinal cortex, contain representations of complex conjunctions of stimulus features, whereas more caudal regions (e.g., V4, TEO) contain the components from which these conjunctions are formed (Barense et al., 2005; Bussey et al., 2002). Damage to the perirhinal cortex compromises the integrity of these complex conjunctive object representations, and thus, as accurate object perception, working memory and long-term memory all require adequate object representations, perirhinal lesions cause deficits in both perception and memory on tasks that necessitate the use of complex feature conjunctions.

In the current study the MTL group did possess more extensive hippocampal and general MTL damage than the hippocampal group, raising the possibility that the poorer performance of the MTL group is due to these volume differences, rather than to perirhinal cortex damage specifically. Although the present findings do not allow us to reject this possibility definitively, there are several reasons why it is unlikely. First, if hippocampal-based memory deficits were responsible for the impairments in the MTL group, one would expect to observe at least a mild deficit in the hippocampal group. This was not the case. Second, the two patient groups were equally impaired on standard measures of recall, and given the severe impairment in recall of both visual and verbal material in the hippocampal group, one cannot argue that the lesions in this group were functionally ineffective. It was on discriminations between objects with a high degree of feature ambiguity that a selective impairment in the MTL group emerged. Finally, dissociations in perirhinal and hippocampal function on object and spatial processing tests have been documented in both neuropsychological (Lee, Bandelow et al., 2006; Lee, Buckley et al., 2006; Lee, Levi, Davies, Hodges, \& Graham, 2007a) and functional neuroimaging (e.g., Barense et al., 2006; Lee, Bandelow et al., 2006; Lee et al., 2007b; Pihlajamaki et al., 2004; Tyler et al., 2004) investigations. For example, patients with semantic dementia, a neurodegenerative condition which affects perirhinal cortex early in the disease (Davies et al., 2004), were impaired on oddity discriminations involving faces, but not scenes (Lee, Bandelow et al., 2006; Lee, Buckley et al., 2006). By contrast, in the same study patients with Alzheimer's disease, who have less involvement of the perirhinal cortex but severe hippocampal damage (Davies et al., 2004), demonstrated difficulties with the scene oddity task, with better performance on face oddity. In support of these findings, recent functional neuroimaging studies have found that high ambiguity discriminations of faces and novel objects (greebles), identical to those used in the present study, were associated with increased activity in the perirhinal cortex, whereas discriminations between scenes were associated with greater hippocampal activity (Barense et al., 2006; Lee et al., 2007b). Thus, several streams of convergent evidence indicate that the perirhinal cortex, but not the hippocampus, is necessary for discriminations of objects with a high degree of feature ambiguity.

It is important to consider why the findings from the present study may have differed from those reported previously. Lee, Bussey et al. (2005) investigated visual discrimination abilities in the same set of MTL patients reported here. Using systematically morphed pairs of stimuli, these authors observed robust impairments in face discrimination, but only mild or non-existent object discrimination impairments. Two followon experiments utilizing a similar procedure to that reported in Lee, Bussey et al. found that individuals with perirhinal damage performed as well as matched controls, particularly on object, compared to face, stimuli (Levy et al., 2005; Shrager et al., 2006). How can one reconcile these findings with the severe object processing deficits reported here? A plausible explanation suggested by the current study is that the degree of deficit elicited on object discrimination tasks is affected by the demand that the task places upon configural processing, or more specifically the demand placed on processing of conjunctions of object features. Thus, it is conceivable that in previous studies using morphed images, participants were able to use a single feature of the blended stimulus (e.g., an eyebrow) to solve the 
task and were never required to process conjunctions of object features.

It is interesting to consider how a role for the perirhinal cortex in processing conjunctions of object features may relate to other cognitive functions that have been attributed to this structure. For example, a large body of evidence suggests that the perirhinal cortex is necessary for familiarity-based memory (e.g., Aggleton \& Brown, 1999). Moreover, it has been demonstrated that familiarity-based memory may be enhanced when subjects are required to process stimulus configurations in an integrative fashion (e.g., processing feature conjunctions as a coherent gestalt) (Giovanello, Keane, \& Verfaellie, 2006; Mandler, Graf, \& Kraft, 1986; Yonelinas, Kroll, Dobbins, \& Soltani, 1999). For example, Yonelinas et al. (1999) demonstrated that familiarity could support associative judgements for faces as long as the different features of the faces were processed holistically. When configural processing was compromised (by presenting the faces upside down), familiarity-based processes no longer contributed to memory performance. Thus, it seems plausible that the feature integration processes assessed in the current experiment may tax a similar perirhinal-based mechanism to the familiarity based associative recognition memory described by Yonelinas et al.

Although our favoured account of these impairments is in terms of object processing (i.e., reflecting activation of an impoverished representation or an inability to store an exact exemplar of a newly presented stimulus), it is important to note that there are alternative explanations that are also consistent with our data. For example, poor object discrimination may emerge, not from perceptual impairments per se, but instead due to an inability to benefit from newly formed long-term memories that may contribute to online object processing (Ryan \& Cohen, 2004). In support of this, Ryan and Cohen (2004) tested participants with amnesia, due to hippocampal or basal forebrain damage, on a scene change detection task with a short (1-2 s) delay. Although patients were unimpaired on most eye-movement measures, they did exhibit a systematic deficit for changes in overall visual sampling of the scene (i.e., the amnesic patients showed no change in overall viewing when shown old versus new scenes). The authors attributed this deficit to the detrimental influence of impaired long-term memory on working memory. In other words, recently processed information is thought to interact with the contents of long-term memory to support working memory performance. This view must presume that working memory is the activated contents of long-term memory (Ericsson \& Kintsch, 1995), but fails to account for the impaired performance of the MTL amnesics reported here on the novel object (greebles and fribbles) tasks - for which subjects would have no pre-existing long-term memory trace.

A related interpretation of the deficits in the current study is that they reflect a deficit in very short-term working memory (i.e., a failure to hold visual information online across saccades between different objects) (Ranganath \& Blumenfeld, 2005). Several recent investigations have directly addressed the status of working memory in amnesia (Hannula et al., 2006; Nichols, Kao, Verfaellie, \& Gabrieli, 2006; Olson, Moore et al., 2006; Olson, Page et al., 2006). These studies report impair- ments in short-term memory for conjunctions of stimuli (e.g., face-scene and object-place conjunctions, and relations of features within a scene). For example, Hannula et al. (2006) tested participants with hippocampal damage on recognition memory for relations among items embedded within scenes and for face-scene pairs. The patients performed poorly in both conditions, even at short lags traditionally considered to be the domain of working memory (i.e., the test probe immediately followed the studied item). Similarly, in an investigation of shortterm working memory following MTL damage, Olson, Page et al. (2006) reported intact memory for individual objects or individual locations, but impaired short-term memory for the conjunction between an object and its presented location at lags as short as one second. Consistent with these findings, a series of fMRI investigations have observed hippocampal involvement on tasks typically considered to assess working memory, such as active maintenance of faces, words or scenes over a short delay (Cabeza, Dolcos, Graham, \& Nyberg, 2002; Karlsgodt, Shirinyan, van Erp, Cohen, \& Cannon, 2005; Nichols et al., 2006; Park et al., 2003; Ranganath \& D'Esposito, 2001). These studies, however, all emphasize the importance of hippocampal contributions to working memory. Any attempt, therefore, to explain the pattern of deficits in the current study according to impairment in working memory is not immediately consistent with the intact performance of the patients with bilateral hippocampal damage reported here. A working memory account must additionally propose that different MTL structures make differential contributions to working memory depending upon the stimuli utilized in the task (e.g., perirhinal cortex supports working memory for complex objects whereas the hippocampus supports working memory for the relationships between objects and their spatial locations).

The working memory hypothesis is difficult to refute: if memory across saccades is considered to be the province of working memory, it is not immediately obvious how one could test perception of complex objects with a large number of overlapping features in the first place. It seems possible, however, that rigid distinctions between psychological constructs such as perception, working memory, and long-term declarative memory may obscure more than they reveal. Indeed, these different processes may be intrinsically interlinked throughout the brain, such that it is impossible to dissociate one from the other. Notably, however, although these views differ according to the proposed cause of the impairment (i.e., compromised working memory versus compromised neural representations), all require a revision to MTL function that extends the role of the MTL beyond long-term memory and takes into account the unique cognitive contribution made by each MTL structure.

Whereas the current findings only tell us what the hippocampus does not do (i.e., object discrimination), there is emerging evidence that the role of the hippocampus can also be extended beyond long-term memory, playing a critical role in creating and storing spatial representations (Burgess, Maguire, \& O'Keefe, 2002; Hartley et al., 2007; Lee, Buckley et al., 2005; Lee, Bussey et al., 2005) and/or in processing representations of relations between perceptually distinct items (e.g., Eichenbaum \& Cohen, 2001; Eichenbaum, Otto, \& Cohen, 1994). For example, using 
a trial-unique virtual-reality scene oddity task identical to the object paradigm reported here, Lee, Buckley et al. demonstrated scene processing impairments in both the patient groups reported in this paper, a pattern that cannot easily be explained without considering a key role for the hippocampus in processing complex spatial layouts independent of long-term declarative memory (although see Hartley et al., 2007). Thus, whereas the present study has shown that the perirhinal cortex - but not the hippocampus - is essential for binding together complex features within individual objects (see also Barense et al., 2005; Bussey et al., 2002), the hippocampus - but not the perirhinal cortex - may be required for binding together information across objects, or more specifically, relational information about objects and their context or spatial location, even over extremely short delays (Burgess et al., 2002; Hannula et al., 2006; Mitchell, Johnson, Raye, \& D'Esposito, 2000; Olson, Page et al., 2006; Ranganath \& Blumenfeld, 2005; Ryan \& Cohen, 2004).

These different MTL structures, therefore, appear to be specialized according to the type of information that they process and are not part of a homogenous system that is exclusively specialized for long-term memory. Taken together, this emerging body of evidence suggests that it may not be particularly useful to draw sharp distinctions between perception, shortterm/working memory and long-term memory, as these neatly segregated parcels of cognitive function may not map onto discrete cortical areas. Instead, a more productive approach in explaining the amnesic syndrome may be to consider the underlying neural representation required to perform the task at hand.

\section{Acknowledgements}

Greebles and fribbles provided courtesy of Michael J. Tarr (Brown University, Providence, RI). We thank Andy Lee and Rhys Davies for help with the anatomical analyses and Ian Nimmo-Smith for statistical assistance. This research was supported by the Medical Research Council, UK and a Peterhouse Research Fellowship to M.D. Barense.

\section{References}

Aggleton, J. P., \& Brown, M. W. (1999). Episodic memory, amnesia, and the hippocampal-anterior thalamic axis. The Behavioural and Brain Sciences, 22(3), 425-444, discussion 444-489.

Barense, M. D., Bussey, T. J., Lee, A. C., Rogers, T. T., Davies, R. R., Saksida, L. M., et al. (2005). Functional specialization in the human medial temporal lobe. Journal of Neuroscience, 25(44), 10239-10246.

Barense, M. D., Lee, A. C. H., \& Graham, K. S. (2006). Medial temporal lobe activation during oddity judgements for objects, faces and scenes. In Paper presented at the Society for Neuroscience Annual Meeting.

Baxter, M. G., \& Murray, E. A. (2001). Opposite relationship of hippocampal and rhinal cortex damage to delayed nonmatching-to-sample deficits in monkeys. Hippocampus, 11(1), 61-71.

Brett, M., Leff, A. P., Rorden, C., \& Ashburner, J. (2001). Spatial normalization of brain images with focal lesions using cost function masking. Neuroimage, 14(2), 486-500.

Buckley, M. J., Booth, M. C., Rolls, E. T., \& Gaffan, D. (2001). Selective perceptual impairments after perirhinal cortex ablation. Journal of Neuroscience, 21(24), 9824-9836.
Buckley, M. J., Charles, D. P., Browning, P. G., \& Gaffan, D. (2004). Learning and retrieval of concurrently presented spatial discrimination tasks: Role of the fornix. Behavourial Neuroscience, 118(1), 138-149.

Buckley, M. J., \& Gaffan, D. (1997). Impairment of visual object-discrimination learning after perirhinal cortex ablation. Behavioral Neuroscience, 111(3), 467-475.

Burgess, N., Maguire, E. A., \& O'Keefe, J. (2002). The human hippocampus and spatial and episodic memory. Neuron, 35(4), 625-641.

Bussey, T. J., \& Saksida, L. M. (2002). The organization of visual object representations: A connectionist model of effects of lesions in perirhinal cortex. European Journal of Neuroscience, 15(2), 355-364.

Bussey, T. J., Saksida, L. M., \& Murray, E. A. (2002). Perirhinal cortex resolves feature ambiguity in complex visual discriminations. European Journal of Neuroscience, 15(2), 365-374.

Cabeza, R., Dolcos, F., Graham, R., \& Nyberg, L. (2002). Similarities and differences in the neural correlates of episodic memory retrieval and working memory. Neuroimage, 16(2), 317-330.

Cave, C. B., \& Squire, L. R. (1992). Intact verbal and nonverbal short-term memory following damage to the human hippocampus. Hippocampus, 2(2), $151-163$.

Davies, R. R., Graham, K. S., Xuereb, J. H., Williams, G. B., \& Hodges, J. R. (2004). The human perirhinal cortex and semantic memory. European Journal of Neuroscience, 20(9), 2441-2446.

Eichenbaum, H., \& Cohen, N. J. (2001). From conditioning to conscious recollection: Memory systems of the brain. New York, NY: Oxford University Press.

Eichenbaum, H., Otto, T., \& Cohen, N. J. (1994). Two functional components of the hippocampal memory system. The Behavioural and Brain Sciences, 17, 449-518.

Ericsson, K. A., \& Kintsch, W. (1995). Long-term working memory. Psychological Review, 102(2), 211-245.

Galton, C. J., Gomez-Anson, B., Antoun, N., Scheltens, P., Patterson, K., Graves, M., et al. (2001). Temporal lobe rating scale: Application to Alzheimer's disease and frontotemporal dementia. Journal of Neurology, Neurosurgery, and Psychiatry, 70(2), 165-173.

Gauthier, I., \& Tarr, M. J. (1997). Becoming a "Greeble" expert: Exploring mechanisms for face recognition. Vision Research, 37(12), 16731682 .

Giovanello, K. S., Keane, M. M., \& Verfaellie, M. (2006). The contribution of familiarity to associative memory in amnesia. Neuropsychologia, 44(10), 1859-1865.

Graham, K. S., Scahill, V. L., Hornberger, M., Barense, M. D., Lee, A. C., Bussey, T. J., et al. (2006). Abnormal categorization and perceptual learning in patients with hippocampal damage. Journal of Neuroscience, 26(29), 7547-7554

Hannula, D. E., Tranel, D., \& Cohen, N. J. (2006). The long and the short of it: Relational memory impairments in amnesia, even at short lags. Journal of Neuroscience, 26(32), 8352-8359.

Hartley, T., Bird, C. M., Chan, D., Cipolotti, L., Husain, M., Vargha-Khadem, F., et al. (2007). The hippocampus is required for short-term topographical memory in humans. Hippocampus, 17(1), 34-48.

Howard, D., \& Patterson, K. (1992). Pyramids and palm trees: A test of semantic access from pictures and words. Bury St. Edmunds, England: Thames Valley Test.

Horel, J. A. (1994). Retrieval of color and form during suppression of temporal cortex with cold. Behavourial Brain Research, 65(2), 165-172.

Insausti, R., Juottonen, K., Soininen, H., Insausti, A. M., Partanen, K., Vainio, P., et al. (1998). MR volumetric analysis of the human entorhinal, perirhinal, and temporopolar cortices. American Journal of Neuroradiology, 19(4), 659-671.

Karlsgodt, K. H., Shirinyan, D., van Erp, T. G., Cohen, M. S., \& Cannon, T. D. (2005). Hippocampal activations during encoding and retrieval in a verbal working memory paradigm. Neuroimage, 25(4), 1224-1231.

Lee, A. C., Bandelow, S., Schwarzbauer, C., Henson, R. N., \& Graham, K. S. (2006). Perirhinal cortex activity during visual object discrimination: An event-related fMRI study. Neuroimage, 33(1), 362-373.

Lee, A. C., Buckley, M. J., Gaffan, D., Emery, T., Hodges, J. R., \& Graham, K. S. (2006). Differentiating the roles of the hippocampus and perirhinal cortex 
in processes beyond long-term declarative memory: A double dissociation in dementia. Journal of Neuroscience, 26(19), 5198-5203.

Lee, A. C. H., Buckley, M. J., Pegman, S. J., Spiers, H., Scahill, V. L., Gaffan, D., et al. (2005). Specialization in the medial temporal lobe for processing of objects and scenes. Hippocampus, 15(6), 782-797.

Lee, A. C. H., Bussey, T. J., Murray, E. A., Saksida, L. M., Epstein, R. A., Kapur, N., et al. (2005). Perceptual deficits in amnesia: Challenging the medial temporal lobe 'mnemonic' view. Neuropsychologia, 43(1), 1-11.

Lee, A. C. H., Levi, N., Davies, R. R., Hodges, J. R., \& Graham, K. S. (2007). Differing profiles of face and scene discrimination deficits in semantic dementia and Alzheimer's disease. Neuropsychologia, 45(9), 2135-2146.

Lee, A. C. H., Scahill, V. L., \& Graham, K. S. (2007b). Activating the medial temporal lobe during oddity judgement for faces and scenes. Cerebral Cortex doi:10.1093/cercor/bhm104 (E-pub).

Levy, D. A., Shrager, Y., \& Squire, L. R. (2005). Intact visual discrimination of complex and feature-ambiguous stimuli in the absence of perirhinal cortex. Learning and Memory, 12(1), 61-66.

Mandler, G., Graf, P., \& Kraft, D. (1986). Activation and elaboration effects in recognition and word priming. The Quarterly Journal of Experimental Psychology, 38A, 645-662.

Mayes, A. R., Holdstock, J. S., Isaac, C. L., Hunkin, N. M., \& Roberts, N. (2002). Relative sparing of item recognition memory in a patient with adult-onset damage limited to the hippocampus. Hippocampus, 12(3), 325-340.

Mitchell, K. J., Johnson, M. K., Raye, C. L., \& D'Esposito, M. (2000). fMRI evidence of age-related hippocampal dysfunction in feature binding in working memory. Brain Research Cognitive Brain Research, 10(1-2), 197-206.

Nelson, H. E. (1976). A modified card sorting test sensitive to frontal lobe defects. Cortex, 12(4), 313-324.

Nichols, E. A., Kao, Y. C., Verfaellie, M., \& Gabrieli, J. D. (2006). Working memory and long-term memory for faces: Evidence from fMRI and global amnesia for involvement of the medial temporal lobes. Hippocampus, 16(7), 604-616.

Olson, I. R., Moore, K. S., Stark, M., \& Chatterjee, A. (2006). Visual working memory is impaired when the medial temporal lobe is damaged. Journal of Cognitive Neuroscience, 18(7), 1087-1097.

Olson, I. R., Page, K., Moore, K. S., Chatterjee, A., \& Verfaellie, M. (2006). Working memory for conjunctions relies on the medial temporal lobe. Journal of Neuroscience, 26(17), 4596-4601.

Osterrieth, P. A. (1944). Le test de copie d'une figure complexe. Archives de Psychologie, 30, 205-220.

Park, D. C., Welsh, R. C., Marshuetz, C., Gutchess, A. H., Mikels, J., Polk, T. A., et al. (2003). Working memory for complex scenes: Age differences in frontal and hippocampal activations. Journal of Cognitive Neuroscience, 15(8), 1122-1134.

Pihlajamaki, M., Tanila, H., Kononen, M., Hanninen, T., Hamalainen, A., Soininen, H., et al. (2004). Visual presentation of novel objects and new spatial arrangements of objects differentially activates the medial temporal lobe subareas in humans. European Journal of Neuroscience, 19(7), 1939-1949.

Raven, J. C. (1962). Coloured progressive matrices sets A, AB, B. London: H.K. Lewis.

Ranganath, C., \& Blumenfeld, R. S. (2005). Doubts about double dissociations between short- and long-term memory. Trends in Cognitive Sciences, 9(8), 374-380.
Ranganath, C., \& D’Esposito, M. (2001). Medial temporal lobe activity associated with active maintenance of novel information. Neuron, 31(5), $865-873$

Rorden, C., \& Brett, M. (2000). Stereotaxic display of brain lesions. Behavourial Neurology, 12(4), 191-200.

Ryan, J. D., \& Cohen, N. J. (2004). The nature of change detection and online representations of scenes. Journal of Experimental Psychology Human Perception and Performance, 30(5), 988-1015.

Saksida, L. M., Bussey, T. J., Buckmaster, C. A., \& Murray, E. A. (2006) No effect of hippocampal lesions on perirhinal cortex-dependent featureambiguous visual discriminations. Hippocampus, 16(4), 421-430.

Scheltens, P., Leys, D., Barkhof, F., Huglo, D., Weinstein, H. C., Vermersch, P., et al. (1992). Atrophy of medial temporal lobes on MRI in "probable" Alzheimer's disease and normal ageing: Diagnostic value and neuropsychological correlates. Journal of Neurology, Neurosurgery, and Psychiatry, 55(10), 967-972.

Seltzer, B., \& Pandya, D. N. (1978). Afferent cortical connections and architectonics of the superior temporal sulcus and surrounding cortex in the rhesus monkey. Brain Research, 149(1), 1-24.

Shallice, T. (1982). Specific impairments of planning. Philosophical Transactions of the Royal Society of London B: Biological Science, 298(1089), 199-209.

Shrager, Y., Gold, J. J., Hopkins, R. O., \& Squire, L. R. (2006). Intact visual perception in memory-impaired patients with medial temporal lobe lesions. Journal of Neuroscience, 26(8), 2235-2240.

Squire, L. R., Shrager, Y., \& Levy, D. A. (2006). Lack of evidence for a role of medial temporal lobe structures in visual perception. Learning and Memory, 13(2), 106-107.

Squire, L. R., Stark, C. E., \& Clark, R. E. (2004). The medial temporal lobe. Annual Review of Neuroscience, 27, 279-306.

Stark, C. E., \& Squire, L. R. (2000). Intact visual perceptual discrimination in humans in the absence of perirhinal cortex. Learning and Memory, 7(5), 273-278.

Suzuki, W. A., \& Amaral, D. G. (1994). Topographic organization of the reciprocal connections between the monkey entorhinal cortex and the perirhinal and parahippocampal cortices. Journal of Neuroscience, 14(3 Pt 2), 1856-1877.

Taylor, K. J., Henson, R. N., \& Graham, K. S. (2007). Recognition memory for faces and scenes in amnesia: Dissociable roles of medial temporal lobe structures. Neuropsychologia, 45(11), 2428-2438.

Tyler, L. K., Stamatakis, E. A., Bright, P., Acres, K., Abdallah, S., Rodd, J. M., et al. (2004). Processing objects at different levels of specificity. Journal of Cognitive Neuroscience, 16(3), 351-362.

Von Bonin, G., \& Bailey, P. (1947). The neocortex of Macaca mulatta. Urbana, IL: University of Illinois.

Warrington, E. K. (1984). The recognition memory test. Windsor: NFER-Nelson.

Warrington, E. K., \& James, M. (1991). Visual object and space perception battery (VOSP). Oxford: Harcourt Assessment.

Williams, P., \& Simons, D. J. (2000). Detecting changes in novel, complex three-dimensional objects. Visual Cognition, 7(1/2/3), 297-322.

Yonelinas, A. P., Kroll, N. E., Dobbins, I. G., \& Soltani, M. (1999). Recognition memory for faces: When familiarity supports associative recognition judgments. Psychonomic Bulletin and Review, 6(4), 654-661. 